\title{
Lessons from Quantile Panel Estimation of the Environmental Kuznets Curve
}

\author{
Carlos A. Flores* \\ Alfonso Flores-Lagunes ${ }^{\dagger}$ \\ Dimitrios Kapetanakis $\ddagger$
}

June 2009

\begin{abstract}
The environmental Kuznets curve (EKC) hypothesizes that the income-pollution relationship has an inverted U shape, implying that pollution is increasing in income up to a turning point beyond which pollution decreases. The empirical literature has concentrated on estimation of this relationship at the mean, employing longitudinal data on countries or U.S. states and measures of per-capita income and emissions of pollutants like $\mathrm{NO}_{x}$ (nitrogen oxide) and $\mathrm{SO}_{2}$ (sulfur dioxide). The typical finding supports an inverted U shaped relationship. Estimation at the mean, however, likely masks heterogeneities that can be present at higher and/or lower quantiles of the emissions' distribution. In addition, mean estimation methods are sensitive to the presence of outliers. This study applies methods for conditional-quantile panel fixed effects models to the estimation of the income-emissions relationship on U.S. state-level data on $\mathrm{NO}_{x}$ and $\mathrm{SO}_{2}$ pollutants over the period 1929-1994. Our results indicate that methods that focus on the conditional mean provide too optimistic estimates about emissions reduction of $N O_{x}$, as conditional-quantile methods suggest that the turning point of the relationship occurs at higher values of income; while the opposite is found for $\mathrm{SO}_{2}$. An important lesson we draw from the application of conditional-quantile techniques is that the income-environmental degradation relationship is sensitive to the presence of outliers in the data.
\end{abstract}

Key words and phrases: Environmental Kuznets Curve, Panel Quantile Estimation, Income and the Environment.

JEL classification: Q56, C21, C23

\footnotetext{
*Department of Economics, University of Miami. Email: caflores@miami.edu

${ }^{\dagger}$ Food and Resource Economics Department, Universty of Florida, and IZA, Bonn, Germany. Email: alfonsofl@ufl.edu

${ }^{\ddagger}$ Food and Resource Economics Department, Universty of Florida. Email: dimitrisk@ufl.edu
} 


\section{Introduction}

What is the relationship between economic development and environmental quality? The Environmental Kuznets Curve (EKC) hypothesis tries to answer this question in terms of the income-pollution relationship. It is graphically represented by an inverted $U$ shape of the income-pollution relationship: at the early stages of a country's development both income and pollution increase, but after a certain level of income is attained, the per capita pollution starts to decrease. The literature estimates the income-emissions relationship typically employing measures of per-capita income and emissions of pollutants such as $N O_{x}$ (nitrogen oxide) and $\mathrm{SO}_{2}$ (sulfur dioxide).

The empirical literature has concentrated on estimation of the EKC at the mean employing longitudinal data on countries or U.S. states (see the recent review by Dinda, 2004). The typical finding is an inverted $\mathrm{U}$ shaped relationship, taken as evidence in favor of the EKC hypothesis. However, the wide range of estimated turning points of this relationship have stimulated a good deal of criticism (e.g. Stern, 2004) and spurred the application of improved methodologies for its estimation, such as semi-parametric and non-parametric methods (e.g., Millimet, List, and Stengos, 2003; Flores, 2007; Zapata, Paudel and Moss, 2008).

Regardless of the methodology employed, however, estimation at the mean likely masks heterogeneities that can be present at higher and/or lower quantiles of the (conditional) emissions' distribution. These potential differences across quantiles are important as ecosystems and humans are more seriously affected at high concentrations of pollutants. In addition, it is well-known that quantile regression is more robust to the presence of outliers, providing an opportunity to examine their effect in the context of the EKC. For these reasons, this study applies methods for conditional-quantile regression estimation of panel fixed effects models (Koenker, 2004 and 2005) to the estimation of the EKC on U.S. state-level data on $N O_{x}$ and $\mathrm{SO}_{2}$ emissions over the period 1929-1994. We employ a specification similar to the one used in previous studies (e.g. List and Gallet, 1999; Millimet, List, and Stengos, 2003) to make our results comparable to them and derive transparent implications from our exercise.

Our results indicate that, for $N O_{x}$, methods that focus on the conditional mean typically provide estimates that are too optimistic about pollution reduction, as conditional-quantile methods reveal that the turning point of the relationship occurs at a higher value of income per-capita, and the reduction in emissions seems to stop toward the high end of income lev- 
els. As for $\mathrm{SO}_{2}$ - where the EKC hypothesis seems to have failed in previous studies (e.g., Millimet, List and Stengos, 2003) — quantile regression offers a slightly more optimistic picture: the relationship is increasing but levels off at a medium level of income and offers prospects of starting a decline. Importantly, the use of quantile regression in each pollutant reveals that very similar shapes of the EKC hold across conditional quantiles. Therefore, our interpretation of the different results with respect to conditional-mean methods is that they are mainly due to the robustness of conditional-quantile methods to outliers in the data.

The reminder of the paper is organized as follows: Section 2 contains a literature review that posits our study in context. Section 3 describes the data and the panel quantile methods employed. In section 4 we discuss the results and their differences from previous results that concentrate in the conditional mean. Section 5 concludes.

\section{Literature Review}

The underpinnings of the EKC hypothesis have been discussed extensively (e.g., de Bruyn and Heintz, 1999; Stern, 2004; Dinda, 2004; Bartz and Kelly, 2008). Most of the work that estimates the income-emissions relationship employs a reduced-form equation that only contains income as an explanatory variable. As a result, these empirical applications do not allow making causal inferences about this relationship. Therefore, the conceptual arguments about the EKC hypothesis are based primarily on theory. We discuss in turn some of the arguments and counter arguments of the EKC hypothesis, followed by the review of some empirical work relevant to our paper.

Arguments in favor EKC hypothesis are as follows: (i) Environmental quality is considered a luxury good having a greater than unit elasticity, such that people in an economy at the early stages of development do not have the luxury of caring about environmental quality. After reaching a level of income at which some basic needs are met, people are willing to pay for a cleaner environment (Selden and Song, 1994). ${ }^{1}$ (ii) As education levels increase with income (a stylized fact), and people with higher education are more aware about the consequences of their economic activities, individuals increase their sensibility about environmental issues. (iii) A more open political system provides the opportunity for people to express their preferences regarding environmental protection. Relative to (i) and (ii), this argument strengthens them

\footnotetext{
${ }^{1}$ Alternatively, even if environmental quality is considered a normal good but the cost of abatement is convex, the inverted U shape implicit in the EKC hypotheis may arise (Kelly, 2003).
} 
since in a more open political system the population's environmental intentions and concerns are revealed through elections. (iv) Economic growth leads to stricter environmental regulation, perhaps through the strengthening of social institutions (Dasgupta et al., 2001). A number of studies note that governments of developed countries impose stricter environmental regulations than the governments of less developed countries (Dinda, 2004). (v) Improvements in technology-which occurs with economic growth-affect environmental degradation either by increasing productivity or by developing processes to reduce specific types of emissions (Stern, 2004; Carrión-Flores and Innes, 2009).

Some of the counter arguments to the EKC hypothesis are as follows: (i) A number of empirical studies (e.g., Flores and Carson, 1995; Komen, Gerkin and Folmer, 1996; Kristom and Riera, 1996) have estimated the elasticity for environmental quality to be smaller than unity. $^{2}$ (ii) Empirical studies that investigate the influence of political systems and civil rights have produced conflicting evidence. For example, Shafik and Bandyopaohyay (1992) find evidence that $\mathrm{SO}_{2}$ concentrations are higher in more democratic countries. (iii) Another counter argument relates to trade liberalization-which increases world production-and the transfer of pollution-intensive industries from developed to developing countries. This transfer may be due to economic specialization whereby manufacturing industries locate in developing countries, or to the developed countries establishing stricter environmental regulations (e.g., see Hettige, Lucas and Wheeler, 1992; Jaffe et al., 1995). Under this argument, environmental improvement might be noticed in developed countries but this is because the pollution has been "exported" to developing countries. Thus, environmental improvement may be just a local phenomenon.

Empirically, early evidence of an inverted U shape in the relationship between certain pollutants and income appeared in Grossman and Krueger (1991), who analyzed the environmental impacts of NAFTA. This study along with Shafik and Bandyopadhyay (1992) popularized the EKC and spurred a large number of follow-up studies (see the surveys in, e.g., De Bruyn and Heintz, 1999; Dinda, 2004). Given the sometimes conflicting evidence reported in empirical studies, researchers have investigated the robustness of the EKC to different data sources, the inclusion of additional control variables, and to the utilization of different econometric techniques that relax various implicit assumptions of the canonical model (Dinda, 2004; Stern, 2004).

\footnotetext{
${ }^{2}$ While these studies may weaken the luxury good assumption, they do not rule out an inverted U shape that could arise through Kelly's (2003) model. See footnote 1.
} 
Studies that use the same data set as we do (a panel of U.S. states) and are thus directly comparable, are List and Gallet (1999), Millimet, List and Stengos (2003), and Flores (2007). The first examines whether income per-capita might have a different effect on emissions in different cross-sectional units, thus estimating different coefficients on income per-capita for each U.S. state. The second study analyzes the issue of functional form misspecification by examining a less restrictive, semiparametric model, while the third considers non-parametric estimation of both the relationship and its turning point. The present study draws insights on the income-emissions relationship by analyzing it at different conditional quantiles of the emissions' distribution.

\section{Data and Methods}

\subsection{Data}

We use panel data at the U.S. state level covering the years 1929-1994 for emissions of nitrogen oxide $\left(\mathrm{NO}_{x}\right)$ and sulfur dioxide $\left(\mathrm{SO}_{2}\right)$. The data contains 48 states and is identical to that originally used in List and Gallet (1999). The data on income comes from the State Annual Summary Tables, 1929-1994, constructed by the U.S. Department of Commerce, while the emissions' data was collected by U.S. Environmental Protection Agency (EPA) and was included in the National Air Pollutant Emission Trends, 1900-1994 report. ${ }^{3}$ In addition to the quality advantage that appears to exist in this particular dataset as discussed in List and Gallet (1999), there are two additional advantages. First, it contains emissions for the entire state and not solely the pollution of urban areas. This feature avoids underestimating pollution, since it is empirically noted that along with development occurs decentralization, which reduces the emissions in urban areas and allocates them to the rest of the region. Second, we do not have to deal with exchange rate issues since we use data from one country. A potential shortcoming of the data is that EPA changed the measurement scheme in 1985 (see Millimet, List and Stengos (2003) for details). However, it has been found that this change in measurement does not result in marked differences in regards to the estimation of the income-emissions relationship (Millimet, List and Stengos, 2003). Given that our results are not qualitatively different from specifications that analyze the two sample periods separately, we concentrate below in an analysis of the full sample 1929-1994. The results for the subsamples are available in Kapetanakis (2009).

\footnotetext{
${ }^{3}$ For details on the data set used see List and Gallet (1999) and Millimet, List and Stengos (2003).
} 


\subsection{Methods}

Following the specification used in most studies to estimate the relationship between emissions and income, we employ a third degree polynomial (Dinda, 2004). This specification is able to capture different possible relationships, such as an inverted U shape, $\mathrm{N}$ shape and monotonically increasing function. As usual in this literature, we work with variables in per-capita terms. The specific functional form used is:

$$
(E / c a p)_{i j}=\alpha_{i}+\gamma_{j}+\beta_{1}(\text { Income } / \text { cap })_{i j}+\beta_{2}(\text { Income } / \text { cap })_{i j}^{2}+\beta_{3}(\text { Income } / \text { cap })_{i j}^{3}+u_{i j}
$$

where $E / c a p$ is emissions per capita, which in our application are given by $\mathrm{NO}_{x}$ or $\mathrm{SO}_{2}$ short tones per capita and represent environmental degradation. Income per capita is measured in 1987 dollars, while $\alpha_{i}$ and $\gamma_{j}$ are state and year fixed effects, respectively. In a first instance, we replicated the fixed effects (FE) models in List and Gallet (1999) and Millimet, List and Stengos (2003) in order to make sure we conduct adequate comparisons with the results from the present study using conditional-quantile regression. In addition, we also estimated-but do not present here-a random effects model which, as in previous studies, is soundly rejected in favor of FE for both pollutants by a Hausman specification test (Hausman, 1978).

The conditional-quantile regression approach to be used in this paper differs from the traditional approach in that it estimates the regression function parameters for different conditional quantiles of the emission's distribution. Thus, instead of estimating the conditional mean equation

$$
E(\mathbf{y} \mid \mathbf{X})=\alpha+\mathbf{X} \boldsymbol{\beta}+u
$$

we estimate

$$
\operatorname{Quant}_{\tau}(\mathbf{y} \mid \mathbf{X}, \tau)=\alpha+\mathbf{X} \boldsymbol{\beta}+u
$$

where $\tau$ is the selected quantile.

The main advantage of considering the model in (3) is that it allows analyzing the incomeemissions relationship at different quantiles of the conditional distribution of emissions. Since the model in (2) only analyzes the mean of the same distribution, quantile regression provides an opportunity to more fully explore the income-emissions relationship. For instance, it is of interest to evaluate whether the EKC hypothesis holds for low and high quantiles of the conditional distribution of emissions. In addition, quantile regression allows for some conditional 
heteroskedasticity in the model (Koenker and Portnoy, 1996), and is a method that is more robust to outliers. ${ }^{4}$

One important practical difference in the estimation of equations (2) and (3) is that they represent different optimization problems. It is well known that the conditional mean model (2) can be estimated by minimizing the mean-squared errors given by the equation

$$
\min _{\beta \in \Re^{p}} \sum_{i=1}^{n}\left(y_{i}-x_{i}^{T} \beta\right)^{2} .
$$

Similarly, Koenker and Basset (1978) provided an estimation method of the conditional quantile in (3) by minimizing

$$
\min _{\beta \in \Re^{p}} \sum_{i=1}^{n} \rho_{\tau}\left(y_{i}-x_{i}^{T} \beta\right)
$$

where $\rho_{\tau}=u(\tau-I(u<0))$ is usually called the check function (Koenker and Basset, 1978; Koenker, 2005).

While estimation methods for the conditional-quantile regression model in (3) are welldeveloped, corresponding methods for panel data-especially $\mathrm{FE}$-have been developed only recently (see Koenker, 2005). The main reason for this is that, although the extension of quantile regression to FE methods is straightforward with data containing a large number of cross-sections and time periods, the typical panel data set with a small number of time series or cross-sections yields the estimation of the multiple FE parameters difficult. The difficulty arises because the method of differencing out fixed effects in the conditional-mean method does not carry over to the quantile method. As a result, penalized methods that shrink the FE coefficients toward a common value have been developed (Koenker, 2004; Lamarche, 2006).

Our data containing 48 U.S. states observed over 66 years, along with the parsimonious reduced-form specification of the relationship, allows us to estimate individual and time fixed effects at each conditional quantile with relatively good precision. This specification is extremely flexible as it allows each conditional quantile to have its own fixed effects. To our knowledge, this is one of the first unrestricted two-way fixed effects quantile regression models estimated. The model we estimate below is given by:

$$
Q_{y_{i j}}\left(\tau \mid x_{i j}\right)=\alpha_{i}(\tau)+\gamma_{j}(\tau)+\mathbf{x}_{i j} \boldsymbol{\beta}(\tau)+u_{i j}
$$

\footnotetext{
${ }^{4}$ See Koenker (2005) for an extensive treatment of quantile regression methods.
} 
where $\mathbf{x}_{i j}$ includes income per-capita, its square and cube; and $\alpha_{i}(\tau)$ and $\gamma_{j}(\tau)$ are the state and the time (year) fixed effects that are allowed to vary across quantiles, respectively. This model dispenses the assumption that one or both types of fixed effects do not depend on the quantile, which is typically imposed when data restrictions (e.g., short panels) require constraining parameters for efficiency purposes. In addition, by avoiding the use of the penalized methods discussed above, we bypass the trade-off of allowing some bias (by shrinking the fixed effects) in order to achieve higher precision. By extension to (5), the minimization problem to solve to estimate the parameters in (6) is given by (Koenker, 2005):

$$
\min _{(\alpha, \beta)} \sum_{k=1}^{q} \sum_{j=1}^{n} \sum_{i=1}^{m_{i}} \rho_{\tau_{k}}\left(y_{i j}-\alpha_{i}\left(\tau_{k}\right)-\gamma_{j}\left(\tau_{k}\right)-x_{i j}^{T} \beta\left(\tau_{k}\right)\right) .
$$

\section{Results}

Table 1 presents descriptive statistics of the variables in our data, including selected quantiles of the unconditional distribution of the variables. Table 2 contains results from the conditionalmean FE model for both pollutants. These results are essentially identical to those from previous studies employing this data (List and Gallet, 1999 and Millimet, List and Stengos, 2003). All coefficients are highly statistically significant. ${ }^{5}$ Given the nature of our study, we concentrate on the graphical appearance of the estimated income-pollution relationship. Figure 1 presents in the first panel the curve calculated from the estimated equation for $N O_{x}$, while the second panel-which employs a different vertical scale-adds to the corresponding curve a scatterplot of the observations in the sample. Both panels have a range of income per capita between zero and $\$ 22,500$ which is approximately the range of this variable in our sample.

From the first panel of Figure 1 it is evident that the conditional-mean FE model offers support to the EKC hypothesis using $N O_{x}$, a result that replicates Millimet, List and Stengos (2003). The turning point for $N O_{x}$ is estimated very precisely to be at $\$ 8,657$ (standard errors obtained via the delta method). From the second panel, there seem to be just a few outlier observations, so they may not have a strong effect on the estimated parameters for this model.

The emissions per capita of $\mathrm{SO}_{2}$ that are presented in the two panels of Figure 2 exhibit a very different behavior. The conditional-mean FE model produces a curve that is monotonically increasing in income per capita (also found in Millimet, List and Stengos, 2003). As a result,

\footnotetext{
${ }^{5}$ As mentioned in section 3.2, a random effects (RE) model is soundly rejected by the data employing a Hausman specification test, presented in Table 2 for each pollutant.
} 
there is no estimated turning point for $\mathrm{SO}_{2}$ and the EKC hypothesis is not supported for this pollutant. In contrast to $N O_{x}$, however, the second panel of Figure 2 clearly shows that there is a larger amount of observations that can be considered outliers, which may be shifting the estimated curve upwards. ${ }^{6}$

Next, we present our conditional quantile regression results for the FE model in (6) for the seven quantiles $\{.05, .1, .25, .5, .75, .90, .95\}$. The estimated parameters (omitting the numerous fixed effects), are presented in Table 3 for each pollutant. They reveal highly statistically significant income per capita coefficients for all quantiles, as well as turning points. ${ }^{7}$ The first panel in Figure 3 plots the estimated curves from the conditional-quantile FE model for $N O_{x}$ for each of the quantiles considered. This figure allows a comparison of the curves across quantiles, which are all very similar in shape, although it is evident that the conditional distribution of $N O_{x}$ is skewed to the right as the upper quantiles are farther away from the median than the lower ones. The figure also allows an examination of the amount of crossings among curves. A large number of curve-crossing is considered an indication of misspecification of the quantile model (Koenker, 2005). Except for a couple of instances (quantiles .5 and .25, and .9 and $.95)$, the curves do not cross despite the polynomial specification of the model that makes the occurrence of crossings more likely for extreme values of the income variable.

The conditional-quantile FE model supports the EKC in the case of $N O_{x}$ (first panel of Figure 3), but it evidences a different curve from the previous conditional-mean FE model. In particular, the rate of decrease when the turning point is attained is much lower compared to the conditional-mean FE model (note that both figures have identical scales). In addition, the conditional-quantile FE model turning points for the different quantiles are in all instances higher compared to the conditional-mean FE turning point. The conditional-quantile turning points range from $\$ 10,282$ to $\$ 11,751$, which are between 19 and 36 percent higher relative to the conditional-mean counterpart. Note also that, at very high levels of income per capita, the conditional-quantile FE model suggests that emissions stabilize and cease to decrease. Finally, the second panel of Figure 3 presents again the scatterplot of the data, which suggests that the

\footnotetext{
${ }^{6}$ Harbaugh, Levinson and Wilson (2002) analyze the effect of outliers on the estimation of the income-emissions relationship. Employing a panel dataset of cities worldwide (that updates the one used by Grossman and Krueger, 1991) and a rule that drops the $5 \%$ of the observations constituting the largest outliers, they find that these outliers have little effect on their results.

${ }^{7}$ All our panel quantile regressions results were obtained using Roger Koenker's R package "quantreg". The package is available at http://cran.r-projrct.org. The standard errors for the coefficients were obtained using the bootstrap option in that package.
} 
conditional-mean FE model is influenced by outliers with low values of emissions at high values of income per capita.

The conditional-quantile FE model curves for the case of $\mathrm{SO}_{2}$ are presented in Figure 4. While it is again evident that the curves do not convincingly support the EKC hypothesis, they offer a slightly more optimistic picture relative to the monotonically increasing relationship estimated from the conditional-mean FE model. The first panel of Figure 4 shows that all of the estimated curves level off at about $\$ 12,000$. In addition, in four out of the seven conditionalquantile FE models estimated $(.05, .25, .75$, and .90) the estimated relationship does exhibit a turning point that ranges from $\$ 13,188$ to $\$ 16,696$ (all statistically significant at conventional levels). Similar to the case of $\mathrm{NO}_{x}$, the $\mathrm{SO}_{2}$ curves show a relative small amount of crossing and have similar shapes in every estimated conditional quantile. In contrast, the conditional distribution of $\mathrm{SO}_{2}$ is not as skewed as that of $\mathrm{NO}_{x}$. The second panel of Figure 4 adds the scatterplot of the data, which suggests that the conditional-quantile FE model is not heavily influenced by outliers with high values of emissions in the case of $\mathrm{SO}_{2}$, relative to the conditionalmean model in Figure 2. In summary, the conditional-quantile FE model presents a more optimistic scenario about the EKC hypothesis for $\mathrm{SO}_{2}$ than does the conditional-mean FE model.

As a final exercise, Figure 5 shows the curves from the conditional-median (0.5 conditional quantile) FE and conditional-mean FE models for each pollutant, along with their corresponding point-wise $95 \%$ confidence intervals computed using the delta method. In the case of $N O_{x}$ in the first panel, we can see that despite the somewhat different curves of the income-emissions relationship predicted in which the conditional-mean model is more optimistic towards the EKC hypothesis, the two curves are not statistically different from each other at conventional levels. A different conclusion is reached for $\mathrm{SO}_{2}$ in the second panel of the figure, as the conditionalmedian FE model produces a curve that is statistically different from the conditional-mean FE model for high levels of income per capita. We attribute this difference to the fact that the conditional-median FE model is more robust to the outliers shown above for this pollutant.

\section{Conclusions}

This study estimates the income-emissions relationship employing conditional-quantile fixed effects (FE) methods. The main advantage of using this methodology is that we are able to 
examine if the Environmental Kuznets Curve (EKC) hypothesis holds at different quantiles of the conditional distribution of emissions. In addition, this methodology is more flexible and robust to outliers in the data relative to conditional-mean methods. We employ a U.S. state-level panel dataset for $\mathrm{NO}_{x}$ and $\mathrm{SO}_{2}$ emissions from the Environmental Protection Agency (EPA) that includes annual observations for 48 states for the time period 1929 to 1994. These data is sufficiently rich that allows the estimation of a very flexible specification of a conditionalquantile panel model that lets the fixed effects (both state and time) to freely vary across the conditional quantiles considered. In order to provide a straightforward comparison of our estimates to conditional-mean FE methods, we employ a cubic polynomial specification for income per capita as in List and Gallet (1999) and Millimet, List, and Stengos (2003).

We find that the estimates from the conditional-quantile FE model provide new insights about the estimation of the income-emissions relationship and the EKC hypothesis. While for $N O_{x}$ emissions both FE models support the EKC hypothesis, the method that focuses on the mean typically provides estimates that are somewhat optimistic about pollution reduction, as conditional-quantile methods suggest that the turning point occurs at income per capita levels that are 19-36 percent higher, and emissions cease to decrease after high levels of income per capita are reached. Nevertheless, when the curves of the income-emissions relationship from conditional median and mean methods are compared, no statistically significant differences arise. Conversely, for $\mathrm{SO}_{2}$-where the EKC hypothesis seems to have failed in previous studies - quantile regression offers a slightly more optimistic picture: the relationship is increasing but levels off at a medium level of income of about $\$ 12,000$. In addition, for four out of seven conditional quantiles investigated we find a turning point (between $\$ 13,188$ and $\$ 16,696$ depending on the quantile) and the curve initiates a slight decline. Given that for both pollutants the use of conditional-quantile FE methods reveals that very similar shapes of the income-emissions relationship hold across conditional quantiles, our interpretation of the different results with respect to conditional-mean methods is that they arise mainly due to the robustness of quantile methods to outliers in the data. Finally, conditional-quantile methods reveal that the conditional distribution of $\mathrm{NO}_{x}$ is skewed to the right, while that of $\mathrm{SO}_{2}$ is more symmetric.

An implication of our results for the estimation of the income-emissions relationship is that the presence of outliers in the typical data available can have important consequences on inference. Therefore, careful examination of these outliers is warranted to determine if they 
are the result of particular activities such as, e.g., the "exporting" of pollution among U.S. states. This is a task we are embarking on within our data. Finally, our results add to the body of literature recognizing that the income-emissions relationship is often not robust to the methodology employed to estimate it. A natural next step in this line of research is to employ even more flexible methodologies, such as the semiparametric quantile panel data procedure of Chen and Khan (2008).

\section{References}

[1] Bartz, S. and D. L. Kelly. 2008. Economic growth and the environment: theory and facts. Resource and Energy Economics, 30: 115-149.

[2] Carrión-Flores, C. E. and R. Innes. 2009. Environmental Innovation and Environmental Performance. Journal of Environmental Economics and Management, forthcoming.

[3] Chen, S. and S. Khan. 2008. Semiparametric estimation of non-stationary censored panel data models with time-varying factor loads. Econometric Theory, 24: 1149-1173.

[4] Dasgupta, S., A. Mody, S. Roy, and D. Wheeler. 2001. Environmental regulation and development: a cross-country empirical analysis. Oxford Development Studies, 29: 173187.

[5] De Bruyn, S. M. and R. J. Heintz. 1999. The environmental Kuznets curve hypothesis. Chapter In Handbook of Environmental and Resource Economics, pp. 656-677. Edward Edgar, Cheltenham.

[6] Dinda, S. 2004. Environmental kuznets curve hypothesis: a survey. Ecological Economics, 49: 431-455.

[7] Flores, C. 2007. Estimation of dose-response functions and optimal doses with a continuous treatment. Discussion paper University of Miami.

[8] Flores, M.E. and R. T. Carson. 1995. The relationship between income elasticities of demand and willingness to pay. Discussion paper University of California San Diego.

[9] Grossman, G.M. and A. Krueger. 1991. Environmental impacts of a north american free trade agreement. Working paper, National Bureau of Economic Research, Cambridge MA: NBER.

[10] Harbaugh,W. T., A. Levinson, and D. M. Wilson. 2002. Reexamining the empirical evidence for an environmental Kuznets curve. Review of Economics and Statistics, 84: 541551.

[11] Hausman, J.A. 1978. Specification tests in econometrics. Econometrica, 46: 1251-1271.

[12] Hettige, H., R. E. B. Lucas, and D. Wheeler. 1992. The toxic intensity of industrial production: global patterns, trends and trade policy. American Economic Review, 82: 478-481.

[13] Jaffe, A., S. Patterson, P. Portney, and R. Stavins. 1995. Environmental regulation and the competitiveness of U.S. manufacturing: what does the evidence tell us. Journal of Economic Literature, 33: 132-163.

[14] Kapetanakis, D. 2009. Lessons from quantile panel regression estimation of the environmental Kuznets curve hypothesis. M.S. Thesis, University of Florida. 
[15] Kelly, D. L. 2003. On environmental Kuznets curve arising from stock externalities. Journal of Economic Dynamics and Control, 27: 1367-1390.

[16] Koenker, R. 2004. Quantile regression for longitudinal data. Journal of Multivariate Analysis, $91: 74-89$.

[17] Koenker, R. 2005. Quantile regression. Cambridge University Press.

[18] Koenker, R. and G. Basset. 1978. Regression quantiles. Econometrica, 46: 33-50.

[19] Koenker, R. and S. Portnoy. 1996. Quentile regression. Working paper No. 97-0100, College of Commerce and Business Administration, University of Illinois at Urbana-Champaign.

[20] Komen, R., S. Gerkin, and H. Folmer. 1996. Income and environmental protection: empirical evidence from OECD countries. Technical report, Department of Economics and Finance, University of Wyoming, Laramie, Laramie, WY.

[21] Kristom, B. and P. Riera. 1996. Is the income elasticity of environmental improvements less than one? Environmental and resource Economics, 7: 45-55.

[22] Lamarche, C. 2006. Robust penalized quantile regression estimator for panel data. Working paper, Department of Economics, University of Oklahoma.

[23] List, J.A. and C. A. Gallet. 1999. The environmental kuznets curve: Does one size fit all? Ecological Economics, 31: 409-424.

[24] Millimet, D.L., J. A. List, and T. Stengos. 2003. The environmental kuznets curve: Real progress or misspecified models? Review of Economics and Statistics, 85:1038-1047.

[25] Panayotou, T. 1997. Demystifying the environmental kuznets curve: Turning a black box into a policy tool. Environment and Development Economics, 2: 465-484.

[26] Selden, T.M and D. Song. 1994. Environmental quality and development: Is there a kuznets curve for air pollution? Journal of Environmental Economics and Management, 27: 147162.

[27] Shafik, N. and S. Bandyopadhyay. 1992. Economic growth and environmental quality: Time series and cross country evidence. Background paper for the world bank report 1992, The World Bank, Washington, DC.

[28] Stern, D. 2004. The rise and fall of the environmental kuznets curve. World Development, 32: $1419-1439$.

[29] Zapata, H., K. Paudel, and C.B. Moss. Functional Form of the Environmental Kuznets Curve. Discussion Paper, Food and Resource Economics Department, University of Florida, 2008. 
Table 1. Descriptive Statistics

\begin{tabular}{lccccccccccc}
\hline Variable & Min & $5^{\text {th }}$ & $10^{\text {th }}$ & 1st Quartile & Median & Mean & 2nd Quartile & $90^{\text {th }}$ & $95^{\text {th }}$ & Max & Std Dev \\
\hline Income & 1,162 & 2,816 & 3,786 & 5,849 & 8,436 & 9,089 & 12,380 & 14,938 & 16,270 & 22,460 & 4,242 \\
$\mathrm{NO}_{x}$ & 0.023 & 0.035 & 0.040 & 0.051 & 0.076 & 0.093 & 0.107 & 0.162 & 0.206 & 1.136 & 0.074 \\
$\mathrm{SO}_{2}$ & 0.002 & 0.025 & 0.036 & 0.059 & 0.097 & 0.165 & 0.184 & 0.335 & 0.544 & 1.618 & 0.206 \\
\hline
\end{tabular}

Income in 1987 dollars, Emissions in thousands short tons

Table 2. Fixed effects model coefficients for $N O_{x}$ and Hausman test statistics

\begin{tabular}{|c|c|c|c|c|}
\hline \multirow[b]{3}{*}{ Coefficient } & \multicolumn{4}{|c|}{ Dependent Variable } \\
\hline & \multicolumn{2}{|c|}{$N O_{x}$} & \multicolumn{2}{|c|}{$\mathrm{SO}_{2}$} \\
\hline & Value & t-value & Value & t-value \\
\hline Income (10e5) & 3.070 & 9.465 & 11.351 & 13.330 \\
\hline Income $^{2}(10 \mathrm{e} 10)$ & -24.034 & -8.241 & -55.873 & -7.296 \\
\hline Income $^{3}(10 \mathrm{e} 15)$ & 48.556 & 5.453 & 107.880 & 4.614 \\
\hline Hausman test $^{a}$ & \multicolumn{2}{|c|}{$\mathrm{p}$-value $=<2.2 \mathrm{e}-16$} & \multicolumn{2}{|c|}{$\mathrm{p}$-value $<2.2 \mathrm{e}-16$} \\
\hline Estimated peak $^{b}$ & \multicolumn{2}{|c|}{$8657(722)$} & \multicolumn{2}{|c|}{-} \\
\hline
\end{tabular}

\section{EKC Fixed Effects for $\mathrm{NO}_{x}$}
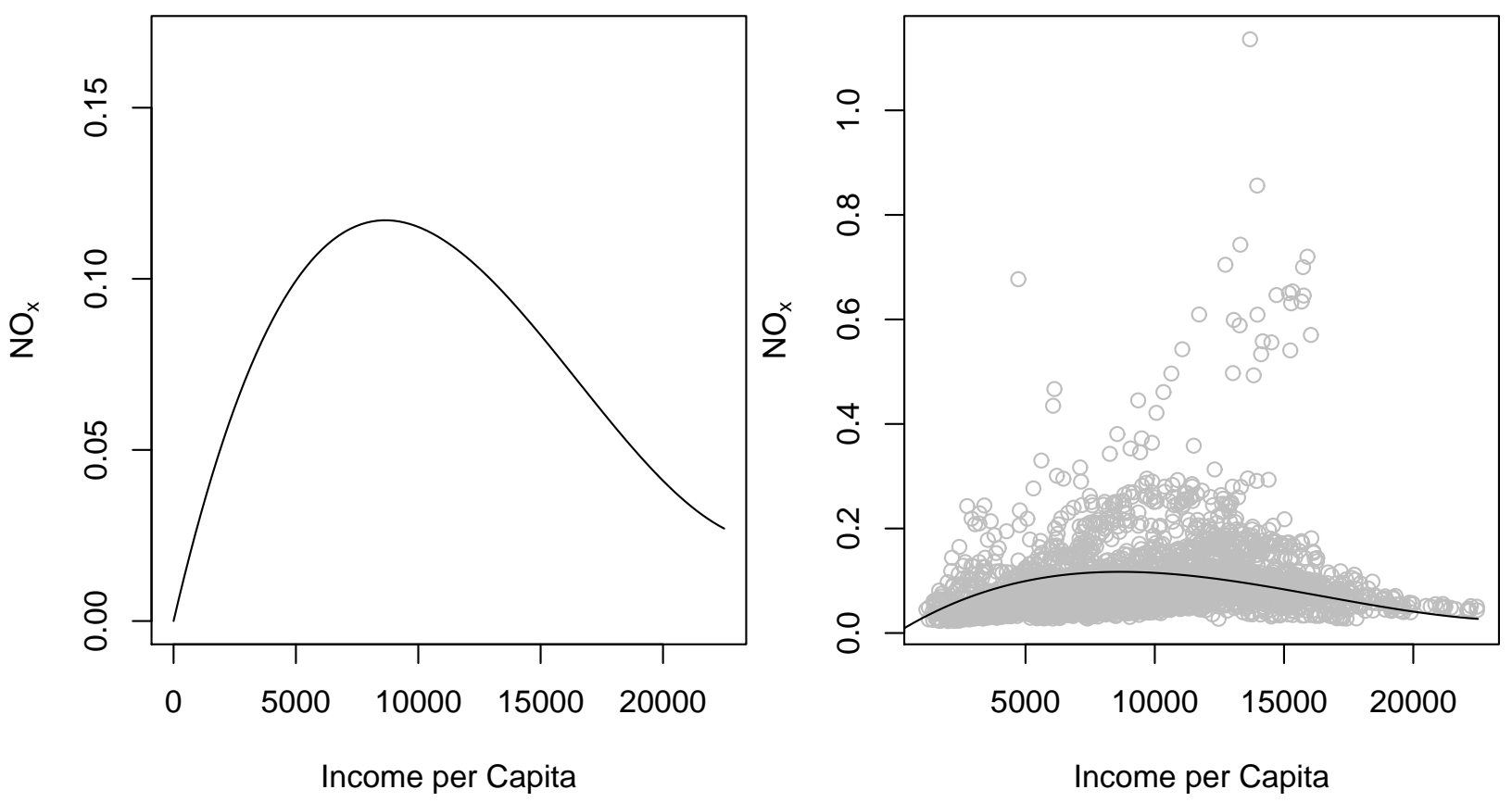

Figure 1. EKC fixed effects for $N O_{x}$ with and without scatterplot 
Table 3. Quantile regression coefficients for $\mathrm{NO}_{x}$ and $\mathrm{SO}_{2}$

\begin{tabular}{|c|c|c|c|c|}
\hline \multirow[b]{3}{*}{ Coefficient } & \multicolumn{4}{|c|}{ Dependent Variable } \\
\hline & \multicolumn{2}{|c|}{$N O_{x}$} & \multicolumn{2}{|l|}{$\mathrm{SO}_{2}$} \\
\hline & Value & $t$ value & Value & $t$ value \\
\hline \multicolumn{5}{|c|}{$5_{t h}$ Percentile } \\
\hline Income (10e5) & 2.286 & 11.142 & 1.817 & 4.595 \\
\hline Income $^{2}(10 \mathrm{e} 10)$ & -15.295 & -10.646 & -10.447 & -3.541 \\
\hline Income $^{3}(10 \mathrm{e} 15)$ & 31.232 & 9.077 & 19.716 & 2.694 \\
\hline Estimated peak ${ }^{a}$ & \multicolumn{2}{|c|}{$11,584(658)$} & \multicolumn{2}{|c|}{$15,472(5,343)$} \\
\hline \multicolumn{5}{|c|}{$10_{t h}$ Percentile } \\
\hline Income (10e5) & 2.409 & 12.660 & 2.374 & 6.261 \\
\hline Income $^{2}(10 \mathrm{e} 10)$ & -16.381 & -12.292 & -12.380 & -4.457 \\
\hline Income $^{3}(10 \mathrm{e} 15)$ & 33.456 & 10.385 & 22.168 & 3.015 \\
\hline Estimated peak & \multicolumn{2}{|c|}{$11,188(609)$} & \multicolumn{2}{|l|}{-} \\
\hline \multicolumn{5}{|c|}{$25_{t h}$ Percentile } \\
\hline Income (10e5) & 2.624 & 15.435 & 3.6049 & 9.361 \\
\hline Income $\left.^{2} 10 \mathrm{e} 10\right)$ & -18.167 & -14.250 & -19.058 & -6.558 \\
\hline Income $^{3}(10 \mathrm{e} 15)$ & 37.786 & 11.362 & 33.148 & 3.964 \\
\hline Estimated peak & \multicolumn{2}{|c|}{$10,991(714)$} & \multicolumn{2}{|c|}{$16,696(4,664)$} \\
\hline \multicolumn{5}{|c|}{ Median regression } \\
\hline Income (10e5) & 2.532 & 15.331 & 4.694 & 12.588 \\
\hline Income $^{2}(10 \mathrm{e} 10)$ & -18.123 & -13.403 & -27.514 & -10.028 \\
\hline Income $\left.^{3} 10 \mathrm{e} 15\right)$ & 37.689 & 9.893 & 55.296 & 6.728 \\
\hline Estimated peak & \multicolumn{2}{|c|}{$10,282(706)$} & \multicolumn{2}{|l|}{-} \\
\hline \multicolumn{5}{|c|}{$75_{t h}$ Percentile } \\
\hline Income (10e5) & 2.635 & 12.042 & 3.6051 & 9.017 \\
\hline Income $^{2}(10 \mathrm{e} 10)$ & -17.829 & -10.360 & -21.299 & -8.115 \\
\hline Income $^{3}(10 \mathrm{e} 15)$ & 34.166 & 7.468 & 38.574 & 5.225 \\
\hline Estimated peak & \multicolumn{2}{|c|}{$10,649(785)$} & \multicolumn{2}{|c|}{$13,188(1,307)$} \\
\hline \multicolumn{5}{|c|}{$90_{t h}$ Percentile } \\
\hline Income (10e5) & 2.298 & 9.163 & $4.4054 e-05$ & 10.891 \\
\hline Income $^{2}(10 \mathrm{e} 10)$ & -14.065 & -7.749 & $-2.5184 e-09$ & -8.056 \\
\hline Income $^{3}(10 \mathrm{e} 15)$ & 24.336 & 5.128 & $4.7417 e-14$ & 5.293 \\
\hline Estimated peak & \multicolumn{2}{|c|}{$11,751(1,033)$} & \multicolumn{2}{|c|}{$15,771(2,327)$} \\
\hline \multicolumn{5}{|c|}{$95_{t h}$ Percentile } \\
\hline Income (10e5) & 2.548 & 8.893 & 4.801 & 10.786 \\
\hline Income $^{2}(10 \mathrm{e} 10)$ & -16.894 & -7.641 & -27.662 & -8.270 \\
\hline Income $^{3}(10 \mathrm{e} 15)$ & 32.481 & 5.497 & 53.210 & 5.614 \\
\hline Estimated peak & 11,089 & $(1,110)$ & - & \\
\hline
\end{tabular}




\section{EKC Fixed Effects for $\mathrm{SO}_{2}$}
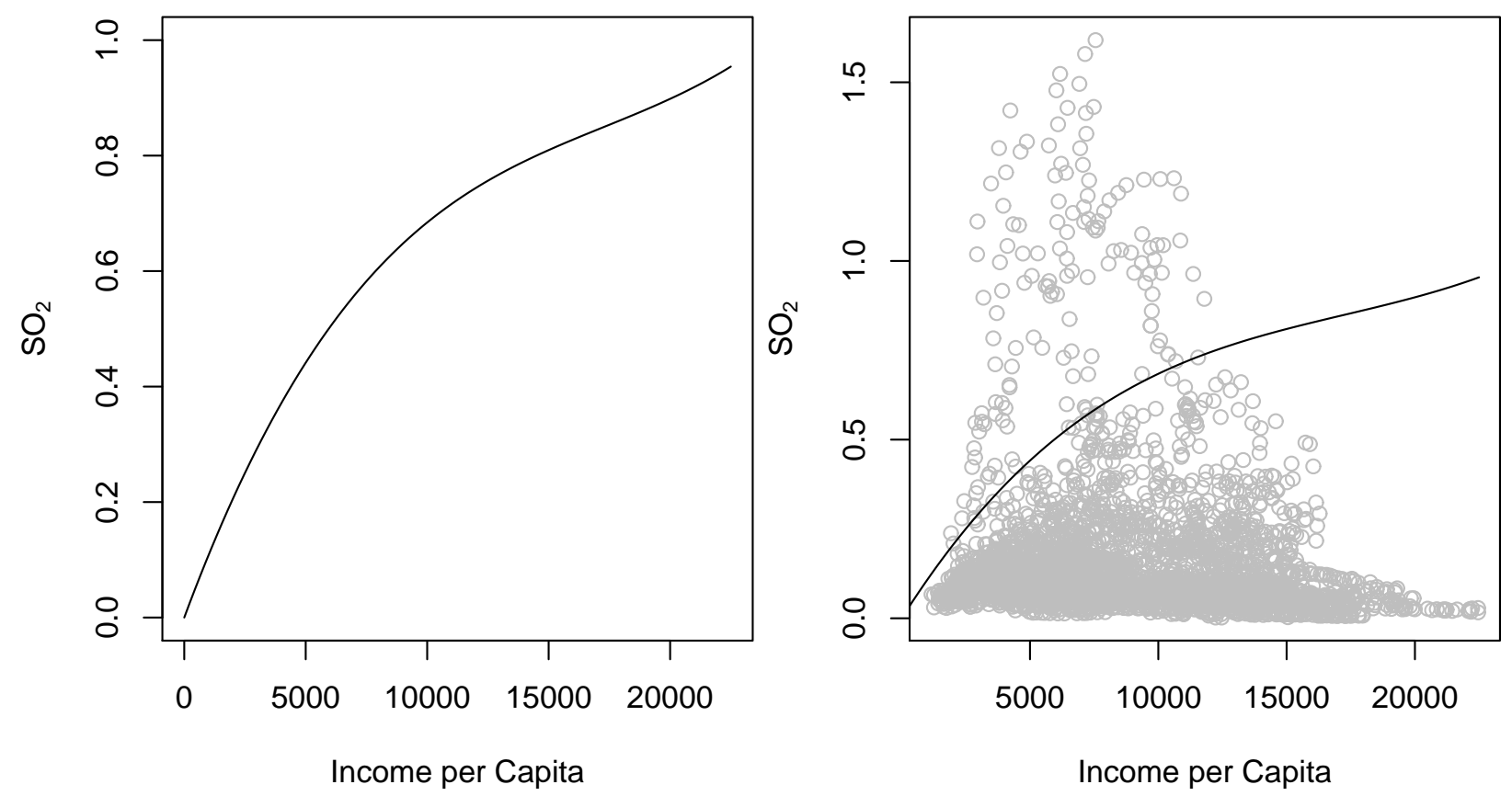

Figure 2. EKC fixed effects for $\mathrm{SO}_{2}$ with and without scatterplot
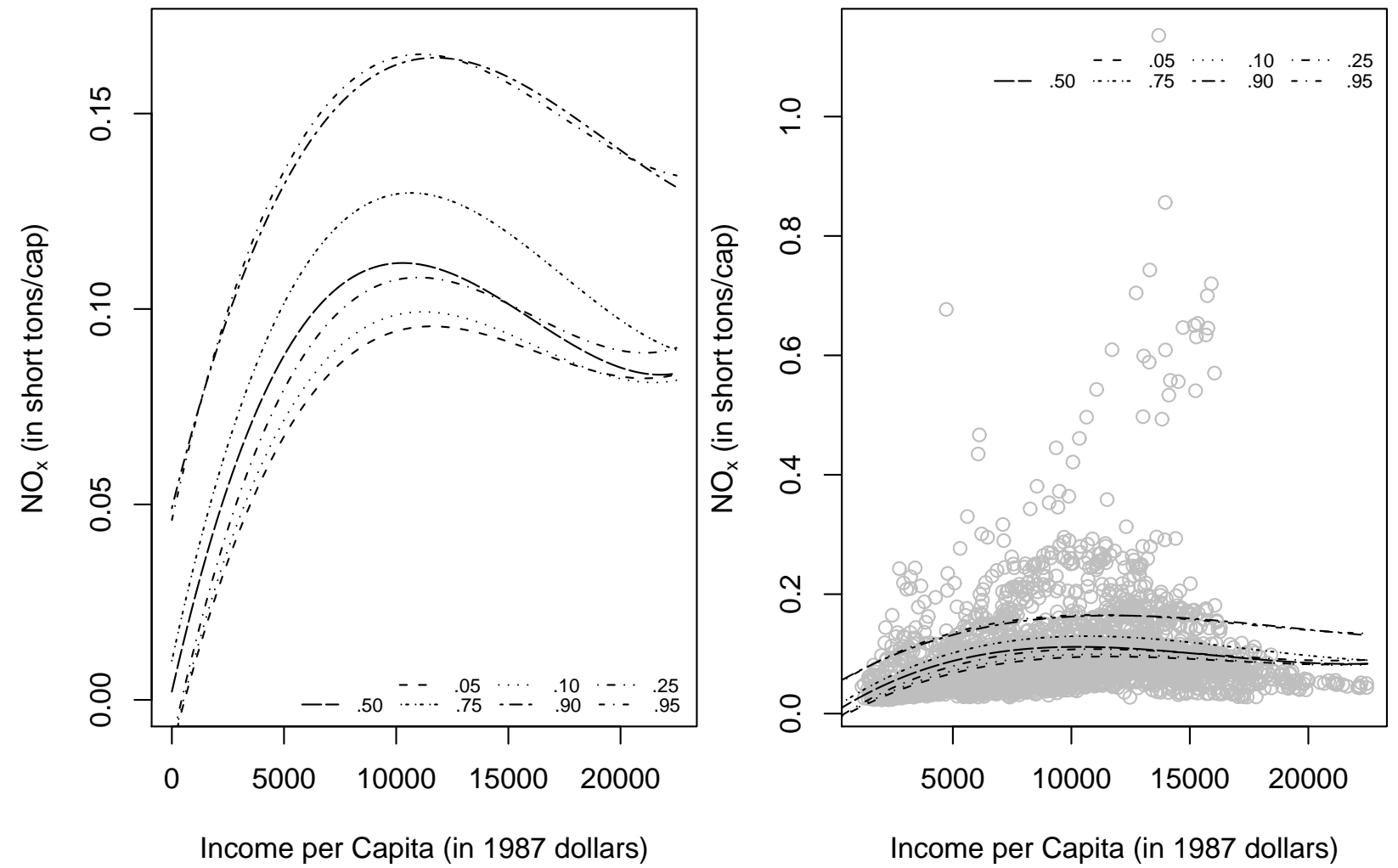

Figure 3. Quantile regression for $N O_{x}$ with and without scatterplot 

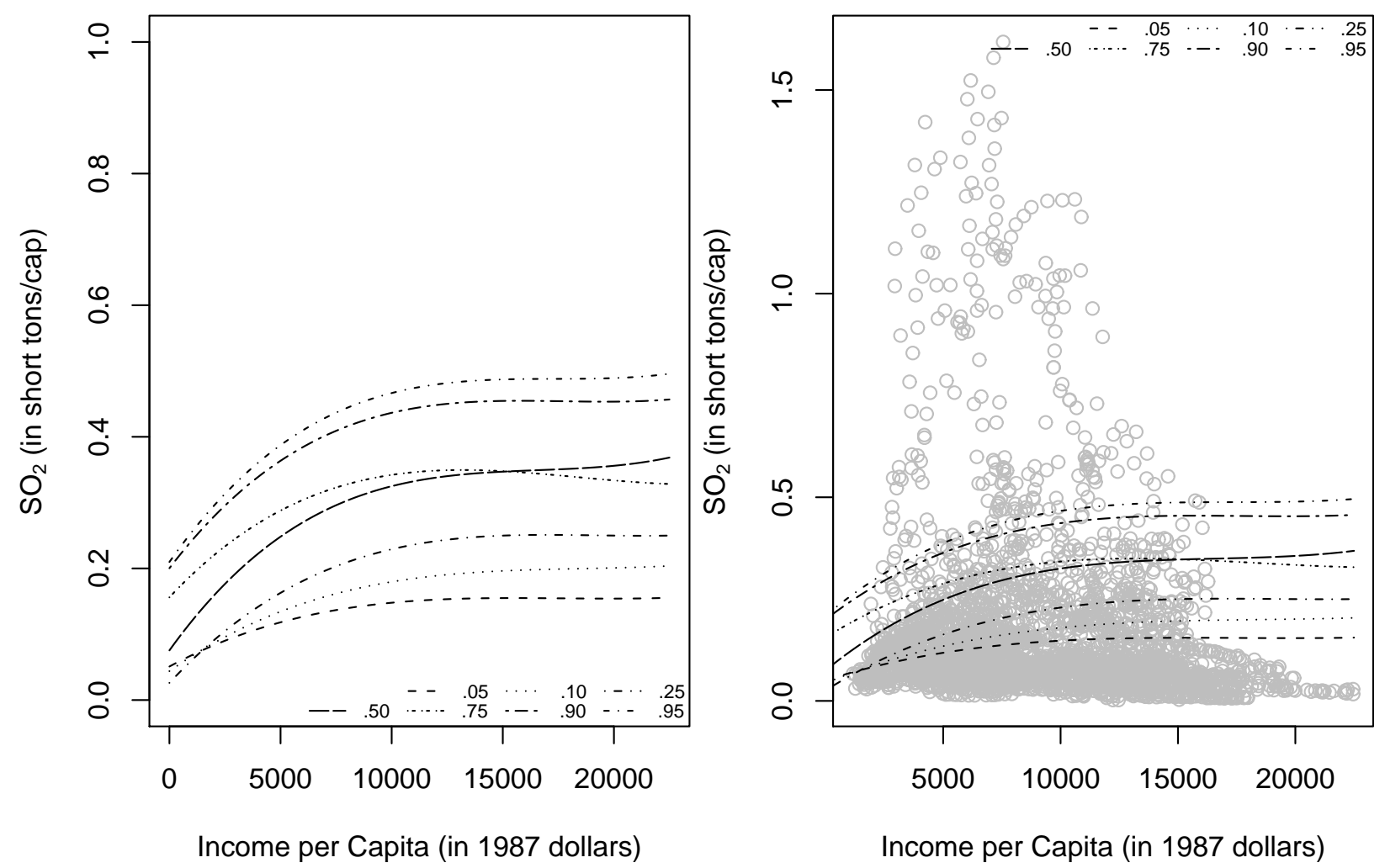

Figure 4. Quantile regression for $\mathrm{SO}_{2}$ with and without scatterplot

\section{Quantile and Mean FE with their Confidence Intervals}

$\mathrm{NO}_{x}$

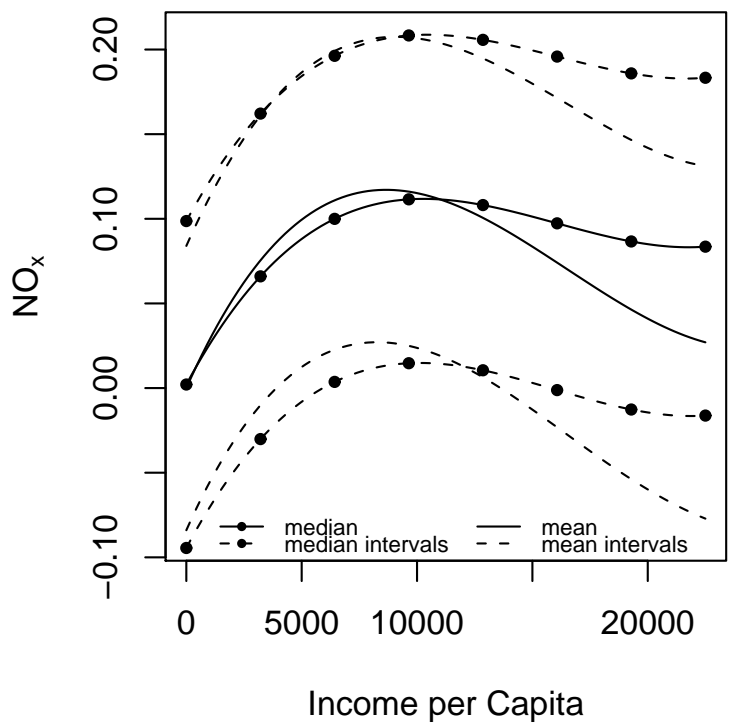

$\mathrm{SO}_{2}$

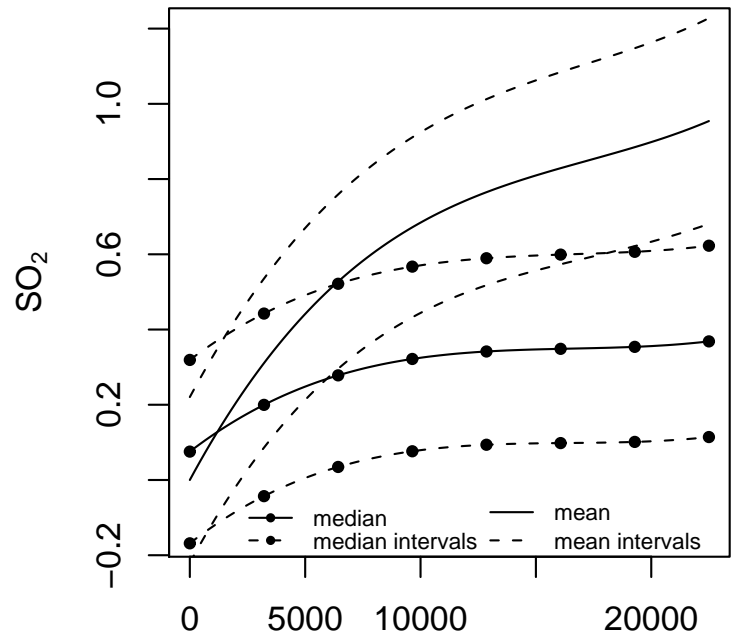

Income per Capita

Figure 5. Curves from median regression and fixed effects model for conditional mean 